\title{
EPR and theoretical studies of positively charged carbon vacancy in $4 \mathrm{H}-\mathrm{SiC}$
}

\author{
T. Umeda and J. Isoya \\ Research Center for Knowledge Communities, University of Tsukuba, Tsukuba 305-8550, Japan \\ N. Morishita, T. Ohshima, and T. Kamiya \\ Japan Atomic Energy Research Institute, Takasaki 370-1292, Japan \\ A. Gali and P. Deák \\ Department of Atomic Physics, Budapest University of Technology and Economics, Budafoki út 8., H-1111, Budapest, Hungary \\ N. T. Son and E. Janzén \\ Department of Physics and Measurement Technology, Linköping University, S-581 83 Linköping, Sweden
}

(Received 6 May 2004; revised manuscript received 20 August 2004; published 22 December 2004)

\begin{abstract}
The carbon vacancy is a dominant defect in $4 \mathrm{H}$-SiC, and the "EI5" electron-paramagnetic-resonance (EPR) spectrum originates from positively charged carbon vacancies $\left(V_{\mathrm{C}}^{+}\right)$at quasicubic sites. The observed state for EI5, however, has been attributed to a motional-averaged state with the $C_{3 \mathrm{v}}$ symmetry, and its true atomic structure has not been revealed so far. We here report low temperature $(<40 \mathrm{~K})$ EPR measurements on EI5 and show that this center has a $C_{1 \mathrm{~h}}$-symmetric structure due to Jahn-Teller distortion. We also performed ab inito calculations of the hyperfine tensors for EI5, and obtained a good agreement between experiment and theory in not only their principal values but also their principal axis directions. A good agreement was also demonstrated for the EI6 center (hexagonal-site $\left.V_{\mathrm{C}}{ }^{+}\right)$in this paper. The transition from $\operatorname{EI} 5\left(C_{1 \mathrm{~h}}\right)$ to $\operatorname{EI} 5\left(C_{3 \mathrm{v}}\right)$ was found to be thermally activated and its activation energy was measured as $0.014 \mathrm{eV}$.
\end{abstract}

DOI: 10.1103/PhysRevB.70.235212

PACS number(s): 61.72.Ji, 76.30.Mi, 71.15.-m

\section{INTRODUCTION}

In silicon carbide $(\mathrm{SiC})$, there are a variety of intrinsic defects not only in radiation-damaged (electron, neutron, ion, etc.) samples but also in as-grown substrates. ${ }^{1}$ These defects are of great importance for the doping efficiency, semiinsulating mechanism, and performance/reliability of $\mathrm{SiC}$ devices. Among dominant intrinsic defects, vacancies of $\mathrm{C}$ or $\mathrm{Si}$, and in particular, $\mathrm{C}$ vacancies $\left(V_{\mathrm{C}}\right)$ are important because of their high thermal stability up to $1000-1500{ }^{\circ} \mathrm{C} .{ }^{1-5} \mathrm{In}$ $4 H$-SiC, their positively charged states give rise to "EI5" and "EI6" electron-paramagnetic-resonance (EPR) spectra, ${ }^{2,3}$ which originate from $V_{\mathrm{C}}{ }^{+}$at quasicubic $(k)$ and hexagonal (h) sites, respectively. ${ }^{4-7}$ The $V_{\mathrm{C}}{ }^{+}$centers at different inequivalent lattice sites [we here denote them as $V_{\mathrm{C}}{ }^{+}(k)$ and $V_{\mathrm{C}}^{+}(h)$ ] exhibited remarkably different Jahn-Teller (JT) distortions. ${ }^{5-7}$ For EI6 or $V_{\mathrm{C}}^{+}(h)$, four surrounding $\mathrm{Si}$ atoms $\left(\mathrm{Si}_{1-4}\right)$ were rearranged to separate $\mathrm{Si}_{1}$ a bit further from $\mathrm{Si}_{2,3,4}$ [Fig. 1(a)], keeping the $C_{3 \mathrm{v}}$ symmetry. ${ }^{5}$ The amount of this distortion, which is manifested by the deviation of $\theta$ [the $\mathrm{Si}_{2,3,4}$ dangling bond (DB) directions, see Fig. 1(a)] from the tetrahedral angle $\left(\approx 109.2^{\circ}\right)$, increased at low temperatures, resulting in a strong temperature dependence of the EI6 spectrum. However, EI5 or $V_{\mathrm{C}}^{+}(k)$ exhibits no largely distorted structure [Fig. 1(b)] and no strong temperature dependence, ${ }^{5}$ despite the $a b$ initio calculations having predicted that EI5 should deform considerably and have a $C_{1 \mathrm{~h}}$-symmetric structure. ${ }^{6}$ Therefore we previously attributed the observed $C_{3 \mathrm{v}}$ configuration for EI5 [Fig. 1(b)] to a motional-averaged state due to thermally activated reorientation. ${ }^{5}$ This was suggested by the fact that below $50 \mathrm{~K}$, the EI5 spectrum became largely complicated and exhibited a lower symmetry. ${ }^{5}$ However, the structure of the low-temperature configuration of EI5 has not been definitely determined so far, because of its complexity.

In this paper, we report a complete analysis of the lowtemperature spectrum of EI5 and show that the EI5 center was distorted to a $C_{1 \mathrm{~h}}$ structure, as consistently with the previous theoretical study. ${ }^{6}$ The EI5 spectrum was measured using the echo-detected EPR technique of pulsed EPR in the $\mathrm{X}$-band $(9-10 \mathrm{GHz})$ range, which made it possible to observe a complete angular dependence of this spectrum free from any disturbances of other signals. We also calculate the hyperfine (HF) tensors including the directions of their principal axes up to the third neighbor atoms $(3 \mathrm{NN})$ of the defects by ab initio supercell methods. The calculated HF tensors and JT distortion for EI5 were found to reproduce very well experimental data. We also found that our calculations fit nicely to the experimental data for EI6 or $V_{\mathrm{C}}{ }^{+}(h)$. The obtained $C_{1 \mathrm{~h}}$ structure for EI5 can explain reasonably the thermally activated reorientation behavior. The activation energy for this reorientation was experimentally estimated to be $0.014 \mathrm{eV}$, which indicates an "electronic bond switching" similar to the cases of vacancies in silicon.

\section{EPR STUDIES}

The EI5 and EI6 centers were created by $3-\mathrm{MeV}$ electron irradiation in commercial $p$-type $4 H-\mathrm{SiC}(0001)$ wafers (room-temperature carrier concentration $\approx 1 \times 10^{15} / \mathrm{cm}^{3}$ ) at $850{ }^{\circ} \mathrm{C}$ with a dose of $4 \times 10^{18}$ electrons $/ \mathrm{cm}^{2}$. This hightemperature irradiation enables us to observe EI5 and EI6 


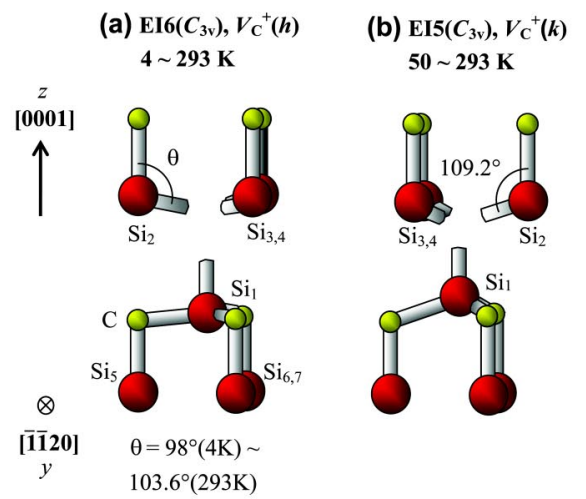

dominantly in the substrates. These substrates were measured by both X-band continuous-wave (cw) EPR (Bruker E500 system) and pulsed-EPR spectrometers (Bruker E580 system). The $\mathrm{X}$-band range was more useful than the $\mathrm{W}$-band $(95 \mathrm{GHz})$ range $^{2,3}$ for measuring the angular dependence of EPR spectra, as will be demonstrated in this paper. At low temperatures, however, usual $\mathrm{cw}-\mathrm{EPR}$ spectra were distorted due to saturation and passage effects. Thus we recorded the EI5 spectra at $5 \mathrm{~K}$ by X-band echo-detected EPR method in which the intensity of the two-pulse Hahn echo ( $\pi / 2$ pulse $-\tau-\pi$ pulse $-\tau$-echo, $\tau=1.2 \mu$ s, the repetition time $=10 \mathrm{~ms}$, the integration window $=1.8 \mu \mathrm{s}$ ) was measured as a function of strength of magnetic field (B). ${ }^{8}$ Since the spin-lattice relaxation time $\left(T_{1}\right)$, phase-memory time $\left(T_{\mathrm{M}}\right)$, and their temperature dependences differ among different centers, the echo-detected spectra consisting of a predominant contribution of EI5 were obtained by selecting the conditions such as the pulse separation, the repetition rate, and the temperature. Pulsed-EPR was also used to measure the spin relaxation times directly in the time domain, ${ }^{8}$ or to determine whether each of the HF lines originates from ${ }^{29} \mathrm{Si}$ or

${ }^{13} \mathrm{C}$ (respective natural abundances are $4.7 \%$ and $1.1 \%$ ) by means of pulsed electron-nuclear-double-resonance (ENDOR) techniques. Pulsed-ENDOR spectra were measured at $5 \mathrm{~K}$ by the Mims sequence $(\pi / 2$ pulse $-\tau-\pi / 2$ pulse $-T-\pi / 2$ pulse $-\tau$-echo, $\tau=0.8 \mu \mathrm{s}, T=16 \mu \mathrm{s}, \mathrm{RF}$ pulse of $10 \mu$ s duration was inserted between the second and the third pulses, the repetition time $=10 \mathrm{~ms}) .^{5}$

Figures 2(a) and 2(b) show angular dependences of EI5 (labeled by bold type) and EI6 spectra (italic typing) measured in the $(\overline{1} 100)$ plane at 150 and $5 \mathrm{~K}$, respectively. At $150 \mathrm{~K}$, both spectra revealed the same $C_{3 \mathrm{v}}$ symmetry with similar angular patterns. ${ }^{29} \mathrm{Si} \mathrm{HF}$ structures of EI5 originate from four $1 \mathrm{NN} \mathrm{Si}$ atoms $\left(\mathrm{Si}_{1}\right.$ and $\left.\mathrm{Si}_{2,3,4}\right)$ of $V_{\mathrm{C}}^{+}$[see Fig. 1(b)]. Also EI6 shows $\mathrm{Si}_{1-4} \mathrm{HF}$ structures (however, $\mathrm{Si}_{1} \mathrm{HF}$ structure is out of sight in Fig. 2). For EI6, since the unpaired-electron distribution concentrates in a $\mathrm{Si}_{1}$ atom by $47 \%,{ }^{5}$ the distribution extends appreciably to $\mathrm{Si}_{5-7}$ atoms located behind $\mathrm{Si}_{1}$, making it possible to observe the $\mathrm{Si}_{5-7} \mathrm{HF}$ structure (see Fig. 2). These characteristics of EI5 and EI6 are fully consistent with the $V_{\mathrm{C}}{ }^{+}$models shown in Fig. 1. At $5 \mathrm{~K}$, however, the angular pattern of EI5 becomes greatly complicated. The central line of EI5 split into three lines, and four pairs of $\mathrm{Si}_{1-4} \mathrm{HF}$ lines split into 12 pairs [see Fig. 2(b)]. In addition, $\mathrm{Si}_{2,3,4} \mathrm{HF}$ lines are separated into two sets of $\mathrm{HF}$ lines at the $c$ axis, which will later be attributed to $\mathrm{Si}_{2}$ and $\mathrm{Si}_{3,4} \mathrm{HF}$ lines, respectively.

In Fig. 2, solid lines show fitted angular patterns for EI5 which were calculated based on the following spin Hamiltonian:

$$
H=\mu_{\mathrm{B}} \mathbf{B} \cdot \mathbf{g} \cdot \mathbf{S}+\Sigma_{n}\left[\mathbf{S} \cdot \mathbf{A}\left(\mathrm{Si}_{n}\right) \cdot \mathbf{I}\left(\mathrm{Si}_{n}\right)-\mu_{\mathrm{N}} g_{\mathrm{N}} \mathbf{I}\left(\mathrm{Si}_{n}\right) \cdot \mathbf{B}\right],
$$

where $\mu_{\mathrm{B}}$ is the Bohr magneton, $\mathbf{g}$ is the $g$ tensor, $\mathbf{S}$ is an electron-spin operator $(S=1 / 2), \mathbf{A}\left(\mathrm{Si}_{n}\right)$ is the HF tensor for the $\mathrm{Si}_{n}$ atom $(n=1-4), \mathbf{I}\left(\mathrm{Si}_{n}\right)$ is a nuclear-spin operator ( $I$ $=1 / 2), \mu_{\mathrm{N}}$ is the nuclear magnetic moment, and $g_{\mathrm{N}}$ is the nuclear $g$ factor for ${ }^{29} \mathrm{Si}\left(g_{\mathrm{N}}=-1.1106\right)$. The fitted lines reproduced very well the experimental data. We could also fit the observed line positions for the rotation of $\mathbf{B}$ in the (11 $\overline{2} 0)$ plane. The spin-Hamiltonian parameters determined from the fits are given in Table I. Using these parameters, we also simulated the angular dependence of the EI5 spectrum at the $\mathrm{W}$ band (Fig. 3). The angular dependence at the $\mathrm{W}$ band is rather difficult to be resolved due to the overlap among all lines, as compared to the case of the X band (Fig. 2). Furthermore, other angular maps such as the EI6's map should fully superimpose on this angular map at the $\mathrm{W}$ band. This fact shows that EPR measurements at higher microwave frequencies are not always superior to the conventional X-band measurements. In Table I, all ${ }^{29} \mathrm{Si} \mathrm{HF}$ tensors are close to be axially symmetric with principal values $A_{\|}$and $A_{\perp}$, where the $A_{\|}$principal axis indicates the direction of Si dangling bonds (DB's). Figure 1(c) shows an atomic model for the lowtemperature state of EI5 constructed from the spinHamiltonian parameters. This structure has $C_{1 \mathrm{~h}}$ symmetry with a $C_{1}$ rotation axis and the $\mathrm{Si}_{3}$ and $\mathrm{Si}_{4}$ atoms being mirror symmetric with respect to the $(11 \overline{2} 0)$ plane. The principal coordinate system of the $g$ tensor is also consistent with this $C_{1 \mathrm{~h}}$ structure. The DB's of $\mathrm{Si}_{1}$ and $\mathrm{Si}_{2}$ atoms are tilted to approach each other, indicating the formation of a $\mathrm{Si}_{1}-\mathrm{Si}_{2}$ atomic pair. Also $\mathrm{Si}_{3}$ and $\mathrm{Si}_{4}$ DB's moved likewise. These behaviors are quite consistent with the theoretical prediction that the pseudo-JT distortion at $V_{\mathrm{C}}{ }^{+}(k)$ induces the $\mathrm{Si}_{1}-\mathrm{Si}_{2}$ and $\mathrm{Si}_{3}-\mathrm{Si}_{4}$ pairs, resulting in the $C_{1 \mathrm{~h}}$ distorted structure. ${ }^{6} \mathrm{In}$ fact, the principal $A$ values indicated an excellent agreement between the experiment and previous theoretical calculation by Bockstedte et al. ${ }^{6}$ (Table I).

This $C_{1 \mathrm{~h}}$ structure can also explain the reorientation effect observed at higher temperatures. Since $\mathrm{Si}_{2,3,4}$ atoms are 


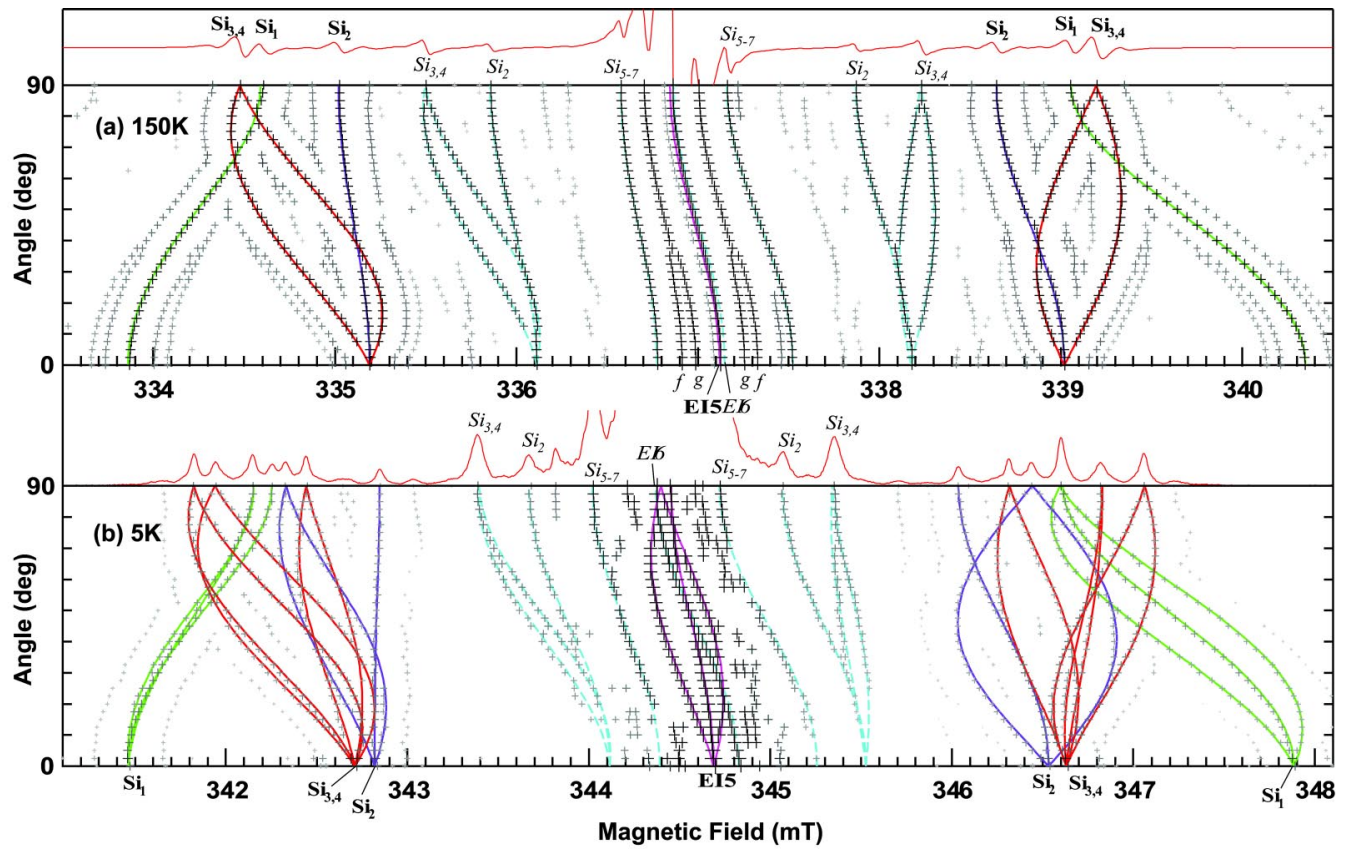

FIG. 2. (Color online) Angular dependence of EI5 and EI6 spectra at (a) $150 \mathrm{~K}$ and (b) $5 \mathrm{~K}$, which were measured by cw EPR $(9.452 \mathrm{GHz})$ and echo-detected EPR $(9.665 \mathrm{GHz})$, respectively. In this figure, magnetic field B was rotated in the $(\overline{1} 100)$ plane from the [0001] $\left(0^{\circ}\right)$ to the $[11 \overline{2} 0]\left(90^{\circ}\right)$ direction, and the spectra for $90^{\circ}$ were displayed. Solid and dashed lines are simulated angular patterns for EI5 and EI6, respectively, using the spin-Hamiltonian parameters in Table I.

$C_{3 \mathrm{v}}$-symmetry related, there should be three equivalent types of atomic-pair formation $\left(\mathrm{Si}_{1}-\mathrm{Si}_{2}, \mathrm{Si}_{1}-\mathrm{Si}_{3}, \mathrm{Si}_{1}-\mathrm{Si}_{4}\right.$ pairs). Thus at higher temperatures when the thermally activated reorientation can occur among the three configuration, the EI5 center will exhibit $C_{3 \mathrm{v}}$ symmetry.

We further examined a dynamics of the reorientation through EPR and pulsed-EPR measurements (Fig. 4). In this figure, low-field-side $\mathrm{Si}_{1-4} \mathrm{HF}$ lines measured for $\mathbf{B} \|[0001]$ are plotted as a function of temperature. The transition from the $C_{3 \mathrm{v}}$ to $C_{1 \mathrm{~h}}$ configuration started at around $40 \mathrm{~K}$, then gradually proceeded, and finally $\mathrm{Si}_{1}, \mathrm{Si}_{3,4}, \mathrm{Si}_{2} \mathrm{HF}$ lines were separated with an intensity ratio of $1: 2: 1$. In this temperature range, it was found that the linewidth of the $\mathrm{Si}_{2,3,4} \mathrm{HF}$ line is strongly correlated to the reorientation. Above $40 \mathrm{~K}$, the linewidth decreased with increasing temperature, indicating the motional narrowing effect. Conversely, below $40 \mathrm{~K}$, the narrowing of $\mathrm{Si}_{3,4}$ and $\mathrm{Si}_{2} \mathrm{HF}$ lines was observed with decreasing temperature, because the characteristic lifetime of the reorientation $(\tau)$ increased and then suppressed the lifetime broadening. These behaviors are common to the reorientation observed in charged vacancies $\left(V^{+} / \mathrm{G} 1 \text { center and } V^{-} / \mathrm{G} 2 \text { center }\right)^{9}$ or a neutral vacancyphosphorous pair $\left[(V+\mathrm{P})^{0}, E \text { center }\right]^{10}$ in silicon. We evaluated $\tau$ by applying the same treatment as the previous works. ${ }^{9-11}$ According to the notations in Ref. 10, we defined

$$
\begin{aligned}
\tau= & p_{A}\left(T_{2}\right)_{B}=p_{B}\left(T_{2}\right)_{A} \quad \text { for lifetime broadening regime, } \\
\tau= & 4 /\left\{\left(T_{2}\right)_{A B} \delta \omega^{2}\right. \\
& \left.\times\left[1-\left(p_{A}-p_{B}\right)^{2}\right]\right\} \text { for motional narrowing regime, }
\end{aligned}
$$

where $T_{2}$ is a transverse spin relaxation time (or phase memory time) of each resonance lines. $A, B$, and $A B$ label for the $\mathrm{Si}_{3,4}, \mathrm{Si}_{2}$, and $\mathrm{Si}_{2,3,4} \mathrm{HF}$ lines, respectively. The relative intensities of $A$ and $B$ are $p_{A}=2 / 3$ and $p_{B}=1 / 3$, and a separation between $A$ and $B$ is $\delta \omega=2.1 \times 10^{7} \mathrm{~s}^{-1}\left[=g \mu_{\mathrm{B}} / \hbar\right.$ $\times 0.12(\mathrm{mT})]$. The $T_{2}$ values were experimentally estimated from two methods. One was deduced from measurements of the cw-EPR linewidth, $\Delta \omega\left(=1 / T_{2}\right)$, where $\Delta \omega$ was estimated by a half width at half maximum of $A, B, A B$ lines minus a static width $(0.032 \mathrm{mT})$. This static width was observed at $5 \mathrm{~K}$. Another method was the direct measurements of $T_{2}$ by two-pulse Hahn echo decay of pulsed EPR. ${ }^{8}$ As shown in the inset of Fig. 3, the data obtained by the two methods coincided excellently. We confirmed that the transition from $A B$ to $A$ and $B$ is triggered when $\tau \approx 1 / \delta \omega\left(4.7 \times 10^{-8} \mathrm{~s}\right)$, as consistent with the original theory. ${ }^{11}$ Also in accordance with our expectation, the lifetime shows a thermally activated behavior (solid line in the figure) and its activation energy $E_{\mathrm{a}}$ was estimated to be approximately $0.014 \mathrm{eV}$. This energy is close to that observed for $V^{ \pm}$in $\mathrm{Si}(0.01$ to $0.02 \mathrm{eV}) .{ }^{9}$ Therefore, as similarly to the case of $\mathrm{Si}$, the reorientation should be caused by "electronic bond switching". ${ }^{9}$ Namely, an unpaired electron just hops between electronic levels of equivalent configurations. This picture is in line with our reorientation model.

It should be noted that similar EPR results have been also obtained in $6 \mathrm{H}$-SiC. ${ }^{12}$ In this polytype, there are two inequivalent $k$ sites $\left(k_{1}, k_{2}\right)$ and two EPR spectra of Ky1 and Ky2 have been assigned to $V_{\mathrm{C}}{ }^{+}\left(k_{1,2}\right)$ (however, the correspondence of each $\mathrm{Ky} 1 / 2$ and each $k_{1 / 2}$ was not given). For $\mathrm{Ky} 2$, the HF tensors of $\mathrm{Si}_{1-4}$ have been obtained (Table I), which are quite close to those of EI5 except smaller $\phi$ of $\mathbf{A}\left(\mathrm{Si}_{3,4}\right)$. Despite the good agreement in the HF tensors be- 
TABLE I. Spin-Hamiltonian parameters for EI5 $\left(C_{1 \mathrm{~h}}\right)$ or $V_{\mathrm{C}}{ }^{+}(k)$ obtained by our EPR experiment and ab initio calculations. Also shown are HF tensors of the previous theoretical calculation (only principal values were reported) (Ref. 6) and EPR results for Ky1/Ky2 centers or $V_{\mathrm{C}}{ }^{+}\left(k_{1,2}\right)$ in $6 H$-SiC (Ref. 12). Principal values of the HF tensors $\left(A_{X, Y, Z}\right)$ are expressed in $\mathrm{mT}$ using a conversion factor that $1 \mathrm{mT}$ $=28.02 \mathrm{MHz}$. We show the principal direction (polar angles $\theta$ and $\phi$ ) only for the main $A_{\|}$axis, because the HF tensors are nearly axial symmetric (see also Fig. 1 for the definition of the coordinate system). The theoretical calculation predicted negative principal values for all HF tensors, however, this table displays only absolute values for comparison with experimental values. $\eta^{2} \alpha^{2}$ and $\eta^{2} \beta^{2}$ indicate $3 s$ - and $3 p$-orbital densities on each $\mathrm{Si}$ atom, estimated from the following relations: $\eta^{2} \alpha^{2}=A_{\text {iso }}(\mathrm{mT}) / 163.93 ; \eta^{2} \beta^{2}=A_{\text {aniso }}(\mathrm{mT}) / 4.08 ; A_{\text {iso }}$ $=\left[A_{\|}\left(\mathrm{Si}_{n}\right)+2 A_{\perp}\left(\mathrm{Si}_{n}\right)\right] / 3 ; A_{\text {aniso }}=\left[A_{\|}\left(\mathrm{Si}_{n}\right)-A_{\perp}\left(\mathrm{Si}_{n}\right)\right] / 3 ; \alpha^{2}+\beta^{2}=1$ (Ref. 5). Finally, a comparison between our experiment and calculation is also demonstrated for EI6 or $V_{\mathrm{C}}{ }^{+}(h)$.

\begin{tabular}{|c|c|c|c|c|c|c|c|c|}
\hline & $X(\perp)$ & $Y(\perp)$ & $Z(\|)$ & $\theta\left(^{\circ}\right)$ & $\phi\left({ }^{\circ}\right)^{\mathrm{a}}$ & $\eta^{2}(\%)$ & $\eta^{2} \alpha^{2}(\%)$ & $\eta^{2} \beta^{2}(\%)$ \\
\hline \multicolumn{9}{|c|}{ EI5 $\left(C_{1 \mathrm{~h}}\right)$ at $5 \mathrm{~K}$, our EPR } \\
\hline g & 2.0056 & 2.0048 & 2.0030 & 22.7 & 0 & & & \\
\hline $\mathbf{A}\left(\mathrm{Si}_{1}\right)$ & 4.44 & 4.36 & 6.46 & 7.7 & 0 & 19.9 & 3.1 & 16.8 \\
\hline $\mathbf{A}\left(\mathrm{Si}_{2}\right)$ & 3.25 & 3.19 & 4.74 & 121.5 & 180 & 14.7 & 2.3 & 12.4 \\
\hline $\mathbf{A}\left(\mathrm{Si}_{3,4}\right)$ & 3.85 & 3.81 & 5.52 & 103.2 & \pm 67.4 & 16.5 & 2.7 & 13.8 \\
\hline \multicolumn{9}{|c|}{$V_{\mathrm{C}}^{+}(k)\left(C_{1 \mathrm{~h}}\right)$, our calculation } \\
\hline $\mathbf{A}\left(\mathrm{Si}_{1}\right)$ & 4.17 & 4.11 & 6.13 & 5.4 & 0 & & & \\
\hline $\mathbf{A}\left(\mathrm{Si}_{2}\right)$ & 3.74 & 3.69 & 5.44 & 117.0 & 180 & & & \\
\hline $\mathbf{A}\left(\mathrm{Si}_{3,4}\right)$ & 3.80 & 3.75 & 5.51 & 105.7 & \pm 61.0 & & & \\
\hline \multicolumn{9}{|c|}{$V_{\mathrm{C}}^{+}(k)\left(C_{1 \mathrm{~h}}\right)$, previous calculation by Bockstedte et al. (Ref. 6) } \\
\hline $\mathbf{A}\left(\mathrm{Si}_{1}\right)$ & 4.35 & 4.07 & 7.03 & & & & & \\
\hline $\mathbf{A}\left(\mathrm{Si}_{2}\right)$ & 3.32 & 3.10 & 5.53 & & & & & \\
\hline $\mathbf{A}\left(\mathrm{Si}_{3,4}\right)$ & 3.89 & 3.68 & 5.74 & & & & & \\
\hline \multicolumn{9}{|c|}{$\mathrm{Ky} 1, \mathrm{Ky} 2$ (both $C_{1 \mathrm{~h}}$ ) in $6 H$-SiC at $4.2 \mathrm{~K}$, EPR by Bratus et al. (Ref. 12) } \\
\hline g $(\mathrm{Ky} 1)$ & 2.0058 & 2.0023 & 2.0025 & 31 & 0 & & & \\
\hline g (Ky2) & 2.0048 & 2.0037 & 2.0022 & 35 & 0 & & & \\
\hline $\mathbf{A}\left(\mathrm{Si}_{1}\right)$ & 4.33 & 4.33 & 6.57 & 7.7 & 0 & 21.4 & 3.1 & 18.3 \\
\hline $\mathbf{A}\left(\mathrm{Si}_{2}\right)$ & 3.16 & 3.16 & 4.71 & 122.0 & 180 & 14.8 & 2.2 & 12.6 \\
\hline $\mathbf{A}\left(\mathrm{Si}_{3,4}\right)$ & 3.93 & 3.93 & 5.59 & 102.7 & \pm 52 & 16.2 & 2.7 & 13.5 \\
\hline \multicolumn{9}{|c|}{ EI6 $\left(C_{3 \mathrm{v}}\right)$ at $5 \mathrm{~K}$, our EPR } \\
\hline g & 2.0026 & 2.0026 & 2.0052 & 0 & 0 & & & \\
\hline $\mathbf{A}\left(\mathrm{Si}_{1}\right)$ & 10.61 & 10.61 & 15.48 & 0 & 0 & 47.3 & 7.5 & 39.8 \\
\hline $\mathbf{A}\left(\mathrm{Si}_{2,3,4}\right)$ & 1.40 & 1.40 & 2.11 & 98 & $0, \pm 120$ & 6.9 & 1.0 & 5.9 \\
\hline $\mathbf{A}\left(\mathrm{Si}_{5,6,7}\right)$ & 0.70 & 0.70 & 0.87 & -16 & $0, \pm 120$ & 1.9 & 0.5 & 1.4 \\
\hline \multicolumn{9}{|c|}{$V_{\mathrm{C}}^{+}(h)\left(C_{3 \mathrm{v}}\right)$, our calculation } \\
\hline $\mathbf{A}\left(\mathrm{Si}_{1}\right)$ & 9.89 & 9.89 & 15.27 & 0 & 0 & & & \\
\hline $\mathbf{A}\left(\mathrm{Si}_{2,3,4}\right)$ & 0.59 & 0.59 & 1.21 & 98.8 & $0, \pm 120$ & & & \\
\hline $\mathbf{A}\left(\mathrm{Si}_{5,6,7}\right)$ & 0.92 & 0.92 & 1.17 & -18.2 & $0, \pm 120$ & & & \\
\hline
\end{tabular}

${ }^{a}$ There is another equivalent orientation for the defect that is given by adding $180^{\circ}$ to this $\phi$ value, because of the symmetry of $4 H$-SiC crystals. For EI6 $\left(C_{3 \mathrm{v}}\right), \phi$ was automatically determined by the restriction of the $C_{3 \mathrm{v}}$ symmetry.

tween EI5 and Ky2, their $g$ tensors are largely different. The reason for this discrepancy may be due to different energyband structures in $4 \mathrm{H}$ - and $6 \mathrm{H}$-SiC and the $g$ tensor reflects not only the wave function but also electronic levels of the defect state.

Using the linear combination of atomic orbital (LCAO) approximation, ${ }^{2,3,5,9,10}$ the unpaired-electron densities $\eta^{2}$ were estimated to be $19.9 \%$ on $\mathrm{Si}_{1}, 14.7 \%$ on $\mathrm{Si}_{2}$, and $16.5 \%$ on $\mathrm{Si}_{3,4}$ (Table I). The unpaired electron distributes equally on the two pairs of $\mathrm{Si}_{1}-\mathrm{Si}_{2}$ and $\mathrm{Si}_{3}-\mathrm{Si}_{4}$. We note that the total value of $\eta^{2}$ for $\mathrm{Si}_{1-4}(67.6 \%)$ is identical to that for EI6 $\left[V_{\mathrm{C}}{ }^{+}(h)\right]$ at $5 \mathrm{~K},{ }^{5}$ because of the same type of origin for EI5 and EI6. Since about $32 \%$ of the defect wave function should extend to $\mathrm{Si}$ or $\mathrm{C}$ atoms located outside $\mathrm{Si}_{1-4}$ atoms, the EI5 spectra clearly revealed super HF structures from such atoms (see Fig. 5). Using pulsed ENDOR, we identified four ${ }^{13} \mathrm{C}$ super HF lines $\left(\mathrm{C}_{\mathrm{I}-\mathrm{IV}}\right)$ and two ${ }^{29} \mathrm{Si}$ super HF lines $\left(\mathrm{Si}_{\mathrm{I}, \mathrm{II}}\right)$. As similarly to the $\mathrm{Si}_{5-7} \mathrm{HF}$ lines for EI6 (see Fig. 2), ${ }^{5}$ we found three $\mathrm{Si}$ atoms $\left(\mathrm{Si}_{\mathrm{I}}\right.$ and $\left.\mathrm{Si}_{\mathrm{II}}\right)$ in the EI5's HF structures which possibly correspond to $\mathrm{Si}_{5}$ and $\mathrm{S}_{6,7}$ atoms of EI5 center, respectively. The split to $\mathrm{Si}_{5}$ and $\mathrm{Si}_{6,7} \mathrm{HF}$ lines is consistent with the $C_{1 \mathrm{~h}}$ symmetry of this center. In the $C_{3 \mathrm{v}}$ configuration observed above $40 \mathrm{~K}$, these super HF structures are averaged out into two HF satellites with the $C_{3 \mathrm{v}}$ symmetry that 


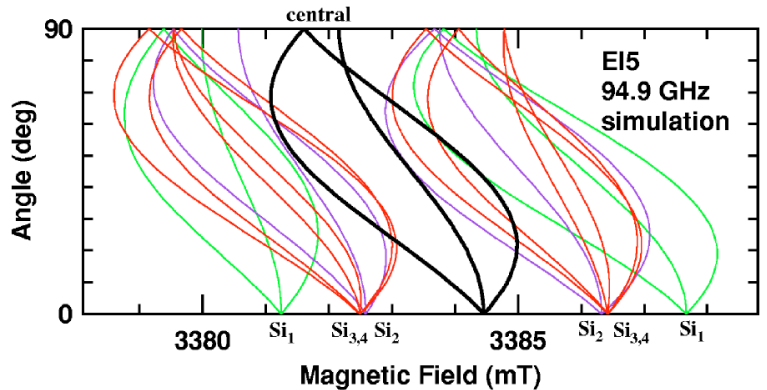

FIG. 3. (Color online) Simulation of angular dependence of EI5 at the $\mathrm{W}$ band $(94.9 \mathrm{GHz})$ in the (1100) plane, using spinHamiltonian parameters in Table I.

have been reported as satellites $f$ and $g$, respectively, in Ref. 5 (and are also seen in Fig. 2).

\section{THEORETICAL CALCULATIONS}

In the previous section, we have seen a good agreement in principal $A$ values for EI5 $\left(C_{1 \mathrm{~h}}\right)$ between our EPR analysis and the previous theoretical work. ${ }^{6}$ However, principal axes of HF tensors are also the key to construct an atomic model of the defect, because these parameters directly indicate a three-dimensional structure of the defect. Therefore we have

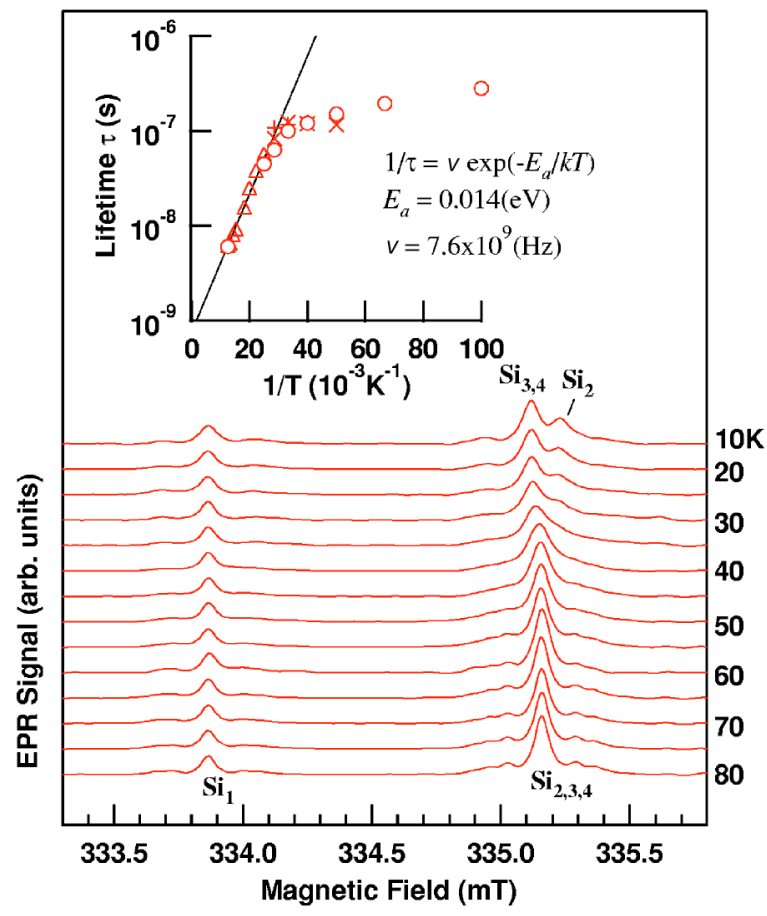

FIG. 4. (Color online) Temperature dependence of ${ }^{29} \mathrm{Si} \mathrm{HF}$ structures for EI5 measured at $9.452 \mathrm{GHz}$. These spectra were obtained by integrating cw-EPR spectra for $\mathbf{B} \|[0001]$. The inset plots the lifetime of the reorientation $(\tau)$ calculated from $T_{2}$. Circles denote the data obtained from direct $T_{2}$ measurements using pulsed EPR, whereas the other symbols (triangles, pluses, and crosses) represent data derived through $\mathrm{cw}$-EPR linewidth measurements for $\mathrm{Si}_{2,3,4}, \mathrm{Si}_{3,4}$, and $\mathrm{Si}_{2} \mathrm{HF}$ lines, respectively. The solid line represents the linear fit using the fitting parameters shown in the figure.

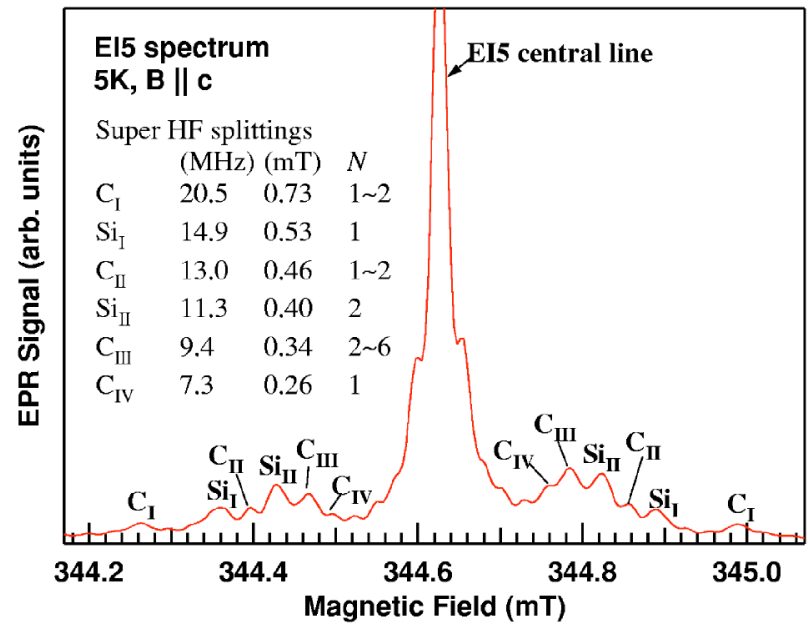

FIG. 5. (Color online) Super HF structures for EI5 $\left(C_{1 \mathrm{~h}}\right)$ measured by echo-detected EPR at $9.665 \mathrm{GHz}$. We have identified their origins ( $\mathrm{Si}$ or $\mathrm{C}$ ) by pulsed ENDOR. Also shown in the figure is the number of atoms corresponding to each super HF line $(N)$, which was deduced from a relative intensity of the HF line.

investigated $V_{\mathrm{C}}^{+}(k)$ and $V_{\mathrm{C}}^{+}(h)$ as well by ab initio quasistatic supercell calculations at $0 \mathrm{~K}$ to verify our models.

We have used a density-functional theory in the localdensity approximation ${ }^{13}$ (DFT-LDA), as implemented in the plane-wave supercell code (FHI98MD) to obtain the optimum geometry. ${ }^{14}$ Ceperley-Alder exchange correlation functional $^{15}$ and norm-conserving Troullier-Martin pseudopotentials ${ }^{16}$ were used. To determine the HF constants of a defect an extremely accurate geometry is required. We have carried out comprehensive convergence tests on the basis size, on the number of relaxed atoms around the defect, on the $K$-point sets, on the shape and size of the supercell for $V_{\mathrm{C}}{ }^{+}(k)$. The minimum-energy configuration was sought by relaxing the atoms around the defect until all the forces were below 0.0005 Hartree/Bohr. According to our calculations, $V_{\mathrm{C}}{ }^{+}$has an electronic configuration of $\left(a_{1}\right)^{2}\left(a_{1}{ }^{\prime}\right)^{1}$ under the crystal field of $4 \mathrm{H}$-SiC. The symmetry of $V_{\mathrm{C}}{ }^{+}$can be kept to be $C_{3 \mathrm{v}}$ or be lowered to $C_{1 \mathrm{~h}}$ due to a pseudo-JT effect involving a nearby $e$ state. $6,7,17$ With constraint to either of these two symmetries we calculated the minimum energy for $V_{\mathrm{C}}{ }^{+}(k)$. We have tested the basis size from $36 \mathrm{Ry}$ up to $64 \mathrm{Ry}$ kinetic energy cutoff, the relaxation of atoms from $2 \mathrm{NN}$ up to $5 \mathrm{NN}$, the $k$-point sets from $1^{3}$ up to $4^{3}$ Monkhorst-Pack (MP) sets, ${ }^{18}$ the supercell size from 72 up to 216 atom supercells. We have found that a 96 atom supercell using a 36 Ry cutoff, $5 \mathrm{NN}$ relaxation, and a $2^{3} \mathrm{MP}$ set provides convergent results for the geometry and energy difference between the $C_{1 \mathrm{~h}}$ or $C_{3 \mathrm{v}}$ symmetry configurations. Accordingly, it was found that the $C_{1 \mathrm{~h}}$ configuration is lower in energy by $0.06 \mathrm{eV}$ than that of the $C_{3 \mathrm{v}}$ configuration which is in line with an earlier finding. ${ }^{6}$ This supports the $C_{1 \mathrm{~h}}$ structure for $V_{\mathrm{C}}{ }^{+}(k)$ at low temperatures. Since the energy differences is much larger than $E_{\mathrm{a}}(=0.014 \mathrm{eV})$ for the thermally activated reorientation, thus the reorientation occurs directly from one $C_{1 \mathrm{~h}}$ configuration to another one, without passing the $C_{3 \mathrm{v}}$ configuration. Our calculations found, however, that $V_{\mathrm{C}}{ }^{+}(h)$ has $C_{3 \mathrm{v}}$ symmetry even at $0 \mathrm{~K}$. To calculate the $\mathrm{HF}$ 
constants of $\mathrm{V}_{\mathrm{C}}^{+}$we have used the supercell code CP-PAW, which is based on the projector augmentation wave method in determining the true wave function close to the nuclei. ${ }^{19}$ We have used the same supercell and MP set as with the FHI98MD code. Two projector functions were used for each angular momentum. The tests on HF constants have shown that even 30 Ry kinetic energy cutoff provides convergent results with using the appropriate projectors.

For $V_{\mathrm{C}}^{+}(k)$, the calculated HF tensors including both principal values and principal axes are compared to the experimental ones in Table I. It is immediately obvious that the calculated and measured HF of the $1 \mathrm{NN} \mathrm{Si}$ atoms $\left(\mathrm{Si}_{1,2,3,4}\right)$ coincide very well in both principal values and axes. For the outer shell of $V_{\mathrm{C}}$, the calculations found that $\mathrm{Si}_{5}$ shows detectable HF interactions (principal values $=0.24,0.27$, $0.45 \mathrm{mT}$ ) and $\mathrm{Si}_{6,7}$ does too (principal values $=0.44,0.45$, $0.59 \mathrm{mT})$. These values are in the same order of the observed HF splittings for $\mathrm{Si}_{\mathrm{I}}\left(\mathrm{Si}_{5}\right)$ and $\mathrm{Si}_{\mathrm{II}}\left(\mathrm{Si}_{6,7}\right)$ (note that the experimental values are not principal values and hence only their orders should be discussed). Among the 12 2NN C atoms around the vacancy, the spin density on eight $\mathrm{C}$ atoms are detectable according to the calculations. That is in line with the experimental observation in Fig. 4.

Finally, we also calculated the principal $A$ values as well as their directions for the case of $V_{\mathrm{C}}^{+}(h)$. The results are summarized in Table I. The calculated HF tensors are again in agreement with the experimental values of EI6 center, even for the $3 \mathrm{NN}$ atoms $\left(\mathrm{Si}_{5,6,7}\right)$, which demonstrates a high reliability of our calculations. In the calculation, the spin density on the first three neighbor $\mathrm{C}$ atoms of $\mathrm{Si}_{1}\left(\mathrm{C}_{\mathrm{I}}\right.$ : principal $A$ values $=0.29,0.31,0.67 \mathrm{mT}$ ) and on one neighbor $\mathrm{C}$ atom for each $\mathrm{Si}_{2-4}$ atom along the $c$ axis $\left(\mathrm{C}_{\mathrm{II}}\right.$ : principal $A$ values $=0.28,0.30,0.50 \mathrm{mT}$ ) were also large, although they have not been observed in experiments. We have found that the HF constants of $3 \mathrm{NN}$ atoms other than $\mathrm{Si}_{5,6,7}$ are below $0.02 \mathrm{mT}$, as consistent with EPR measurements.

\section{SUMMARY}

We have revealed the $C_{1 \mathrm{~h}}$-symmetric atomic structure for the EI5 center $\left[V_{\mathrm{C}}^{+}(k)\right]$, which was observable below $40 \mathrm{~K}$, by means of the optimized X-band echo-detected EPR measurements. This structure was characterized by pairing of four $\mathrm{Si}$ atoms $\left(\mathrm{Si}_{1}-\mathrm{Si}_{2}\right.$ and $\left.\mathrm{Si}_{3}-\mathrm{Si}_{4}\right)$ of $V_{\mathrm{C}}$ due to JT distortion. We also carried out the first-principle calculations and demonstrated that the HF tensors (both principal values and axes) for $V_{\mathrm{C}}{ }^{+}(k)$ were in good agreement with those in our EPR experiments. Also this paper demonstrated a good correspondence between the EI6 center and theoretical calculation for $V_{\mathrm{C}}^{+}(h)$, ensuring the reliability of our calculations. Above $40 \mathrm{~K}$, the EI5 center transformed from the $C_{1 \mathrm{~h}}$-symmetric center to the $C_{3 \mathrm{v}}$-symmetric one. This phenomenon was attributed to a thermally activated reorientation of $V_{\mathrm{C}}{ }^{+}(k)$. The presence of such reorientation was consistent with the atomic structure of $\mathrm{V}_{\mathrm{C}}^{+}(k)$ determined here. The activation energy for this reorientation was found to be $0.014 \mathrm{eV}$, which indicated "electronic bond switching" behavior the same as observed for vacancies in silicon.
${ }^{1}$ For example, N. T. Son, B. Magnusson, Z. Zolnai, A. Ellison, and E. Janzén, Mater. Sci. Forum 457-460, 437 (2004).

${ }^{2}$ N. T. Son, P. N. Hai, and E. Janzén, Phys. Rev. B 63, 201201(R) (2001).

${ }^{3}$ N. T. Son, P. N. Hai, and E. Janzén, Phys. Rev. Lett. 87, 045502 (2001).

${ }^{4}$ Z. Zolnai, N. T. Son, B. Magnusson, C. Hallin, and E. Janzén, Mater. Sci. Forum 457-460, 473 (2004).

${ }^{5}$ T. Umeda, J. Isoya, N. Morishita, T. Ohshima, and T. Kamiya, Phys. Rev. B 69, 121201(R) (2004).

${ }^{6}$ M. Bockstedte, M. Heid, and O. Pankratov, Phys. Rev. B 67, 193102 (2003).

${ }^{7}$ A. Gali, P. Deák, N. T. Son, and E. Janzén, Mater. Sci. Forum 433-436, 511 (2003).

${ }^{8}$ J. Isoya, S. Yamasaki, H. Okushi, A. Matsuda, and K. Tanaka, Phys. Rev. B 47, 7013 (1993).
${ }^{9}$ G. D. Watkins, Radiation Effects in Semiconductors (Plenum Press, New York, 1968).

${ }^{10}$ G. D. Watkins and J. W. Corbett, Phys. Rev. 134, A1359 (1964).

${ }^{11}$ H. S. Gutowsky and A. Saika, J. Chem. Phys. 21, 1688 (1953).

${ }^{12}$ V. Ya. Bratus, I. N. Makeeva, S. M. Okulov, T. L. Petrenko, T. T. Petrenko, and H. J. von Bardeleben, Physica B 308-310, 621 (2001).

${ }^{13}$ W. Kohn and L. J. Sham, Phys. Rev. 140, A1133 (1965).

${ }^{14}$ M. Bockstedte, A. Kley, J. Neugebauer, and M. Scheffler, Comput. Phys. Commun. 107, 187 (1997).

${ }^{15}$ D. M. Ceperley and B. J. Alder, Phys. Rev. Lett. 45, 566 (1980).

${ }^{16}$ N. Troullier and J. L. Martins, Phys. Rev. B 43, 1993 (1991).

${ }^{17}$ A. Zywietz, J. Furthmüller, and F. Bechstedt, Phys. Rev. B 59, 15166 (1999).

${ }^{18}$ H. J. Monkhorst and J. K. Pack, Phys. Rev. B 13, 5188 (1976).

${ }^{19}$ P. E. Blöchl, Phys. Rev. B 62, 6158 (2000). 